\title{
Structure of freely suspended chiral smectic films as determined by x-ray reflectivity and optical ellipsometry
}

\author{
A. Fera, R. Opitz, and W. H. de Jeu \\ FOM-Institute for Atomic and Molecular Physics, Kruislaan 407, I098 SJ Amsterdam, The Netherlands \\ B. I. Ostrovskii \\ Institute of Crystallography, Academy of Science of Russia, Leninski pr. 59, Moscow 117333, Russia \\ D. Schlauf and Ch. Bahr \\ Institute of Physical Chemistry, University Marburg, D-35032 Marburg, Germany
}

(Received 17 January 2001; published 17 July 2001)

\begin{abstract}
We report on a combined x-ray reflectivity and optical ellipsometry study of freely suspended smectic $(\mathrm{Sm})$ films of a chiral liquid crystalline compound with the phase sequence $\mathrm{Sm}-A-\mathrm{Sm}-\mathrm{C}_{\alpha}^{*}-\mathrm{Sm}-\mathrm{C}^{*}-$ $\mathrm{Sm}-\mathrm{C}_{\gamma}^{*}-\mathrm{Sm}-\mathrm{C}_{A}^{*}$. Using tilt magnitude profiles from $\mathrm{x}$-ray reflectivity as input to model the average optical properties obtained by ellipsometry, tilt direction profiles are also obtained. In this way realistic models can be elaborated for the various types of chiral Sm-C films. We find that the surface layers are more tilted than the interior layers due to surface interactions and finite size effects. For the ferrielectric $\mathrm{Sm}-\mathrm{C}_{\gamma}^{*}$ phase the tilt direction profile corresponds to a three-layer helix, in agreement with the clock model of chiral Sm-C phases. In thin films the surface interactions suppress the bulk helix structure of the $\mathrm{Sm}-\mathrm{C}_{\alpha}^{*}$ phase and a Sm- $\mathrm{C}_{A}^{*}$-like structure is formed with an anticlinic layer-by-layer alternation of the tilt directions.
\end{abstract}

DOI: 10.1103/PhysRevE.64.021702

PACS number(s): 61.30.Eb, 64.70.Md, 78.70.Ck

\section{INTRODUCTION}

The structure and origin of the polar behavior of tilted smectic liquid crystals formed by chiral molecules have been intensively debated over the last decade $[1,2]$. Smectic liquid crystals consist of stacks of molecular layers, each of which form a two-dimensional (2D) liquid. Apart from the smectic- $A$ phase $(\mathrm{Sm}-A)$ in which the director $\mathbf{n}$ (the local preferred direction of the long molecular axes) is normal to the layer plane, a variety of tilted phases exist in which $\mathbf{n}$ is inclined by an angle $\theta$ with respect to the layer normal $\mathbf{z}$. The azimuthal orientation of the director about $\mathbf{z}$ is defined by an angle $\varphi(z)$. In the classical smectic-C phase (Sm-C) the azimuthal orientations in adjacent layers are in the same direction: $\varphi(z)$ is constant. Such a synclinic ordering leads to ferroelectricity in a Sm-C phase of chiral molecules $\left(\mathrm{Sm}-\mathrm{C}^{*}\right)$ : the symmetry allows in each layer for a spontaneous electric polarization $\mathbf{P}$ along $\mathbf{n} \times \mathbf{z}$ (perpendicular to the tilt plane) [3]. In addition the molecular chirality generates a helical twist with a pitch of the order of microns due to the azimuthal angle varying slowly from one layer to another. Since this first discovery a whole family of chiral tilted smectic phases has been found. For opposite tilt directions of adjacent layers [4], the resulting anticlinic ordering corresponds to an antiferroelectric $\mathrm{Sm}-\mathrm{C}_{A}^{*}$ phase in which the direction of the polarization alternates from layer to layer. Also tilted phases with ferrielectric $\left(\mathrm{Sm}-\mathrm{C}_{\gamma}^{*}\right)$ and mixed antiferroelectric-ferrielectric $\left(\mathrm{Sm}-\mathrm{C}_{\alpha}^{*}\right)$ properties have been identified [2]. On the temperature scale the $\mathrm{Sm}-\mathrm{C}_{\alpha}^{*}$ phase appears usually between the $\mathrm{Sm}-A$ and the $\mathrm{Sm}-\mathrm{C}^{*}$ phase [5]. The director field in the $\mathrm{Sm}-\mathrm{C}_{\gamma}^{*}$ and the $\mathrm{Sm}-\mathrm{C}_{\alpha}^{*}$ phase is characterized by a complex distribution of the molecular tilt, and the exact interlayer structure of these phases is still sub- ject to debate. A first structural model is based on the 1D Ising model $[2,6]$. It predicts an infinitely large number of structural states ("devil's staircase") characterized by various sequences of synclinic/anticlinic interfaces. Alternative models are based on a discrete variation of the azimuthal angle $\varphi$ across the layers ("clock models") [7]. Resonant $\mathrm{x}$-ray scattering provided direct evidence of three-layer and four-layer superlattices in the ferrielectric phases, which is consistent with the clock model [8]. However, recent ellipsometric measurements rule out a simple uniaxial clock model for ferrielectric phases and favor a highly distorted biaxial mode [9]. For the $\mathrm{Sm}-\mathrm{C}_{\alpha}^{*}$ phase resonant x-ray scattering indicates an incommensurate periodicity, varying from five to eight layers [8], which has been confirmed by ellipsometry $[10,11]$ and optical reflectivity [11].

The picture sketched so far is in practice more complicated because the ordering in liquid crystals is strongly influenced by finite size and surface effects. A free surface may often stabilize a higher ordered phase that is either observed in the bulk at lower temperatures or not observed in the bulk at all [12]. Such experiments can be carried out advantageously with freely suspended smectic films. Due to their controlled thickness (from two to over hundreds of layers) and the uniform ordering of the smectic layers parallel to the surfaces, these films provide perfect model system to study phase transformations and surface induced ordering in substrate-free finite-size systems [13]. In the case of a $\mathrm{Sm}-A-\mathrm{Sm}-\mathrm{C}$ transition a free surface often induces a tilt, causing the Sm-C phase to grow continuously from the surface into the $\mathrm{Sm}-A$ interior of the film as the $A \mathrm{C}$ transition temperature is approached from above [14]. As a consequence a tilt magnitude profile $\theta(z)$ appears across the film. For chiral Sm-C films we can expect this to be accompanied by a tilt direction profile $\varphi(z)$. 


\section{$\mathbf{F}$ $\mathrm{C}_{12} \mathrm{H}_{25} \mathrm{O}-\mathrm{O}-\mathrm{O}-\mathrm{COO}-\mathrm{O}-\mathrm{COO}-\mathrm{CH}\left(\mathrm{CH}_{3}\right)-\mathrm{C}_{6} \mathrm{H}_{13}$ $\mathrm{I} \stackrel{106}{\leftrightarrow} \mathrm{Sm}-\mathrm{A} \stackrel{97}{\leftrightarrow} \mathrm{Sm}-\mathrm{C}_{\alpha}^{*} \stackrel{92}{\leftrightarrow} \mathrm{Sm}-\mathrm{C}^{*} \stackrel{83}{\leftrightarrow} \mathrm{Sm}-\mathrm{C}_{\gamma}^{*} \stackrel{78}{\leftrightarrow} \mathrm{Sm}_{-} \mathrm{C}_{\mathrm{A}}^{*}$}

FIG. 1. Molecular formula of compound 12F1M7 and the transition temperatures $\left({ }^{\circ} \mathrm{C}\right)$ for a 40 -layer film.

$\mathrm{X}$-ray reflectivity has been extensively used to characterize the structure and interfacial properties of hard- and softmatter thin films [15]. Specular reflectivity probes the average electron density $\rho(z)$ along the surface normal, which allows deriving tilt magnitude profiles. Ellipsometry is sensitive to the optical ellipsoid of refraction (optical indicatrics) averaged over the film [16]. This gives the mean tilt angle $\langle\theta\rangle$ and also allows distinguishing between ferroelectric and antiferroelectric states [17]. By combining both methods the tilt magnitude profile $\theta(z)$ from x-ray reflectivity can be used as input for modeling the average optical quantities obtained by ellipsometry. This allows elaborating realistic structural models of the various types of $\mathrm{Sm}-\mathrm{C}^{*}$ phase in thin films and in particular to obtain also tilt direction profiles $\varphi(z)$.

In this paper we present results from a combined x-ray reflectivity and optical ellipsometry study of a chiral compound with the phase sequence $\mathrm{Sm}-A-\mathrm{Sm}-\mathrm{C}_{\alpha}^{*}-$ $\mathrm{Sm}-\mathrm{C}^{*}-\mathrm{Sm}-\mathrm{C}_{\gamma}^{*}-\mathrm{Sm}-\mathrm{C}_{A}^{*}$. Within the accuracy of our measurements there are no indications of subharmonics of the single layer periodicity. This signifies that all the layer interfaces are identical, and provides strong independent support for the clock model for the tilt direction distribution in the various chiral Sm-C phases. We show that surface interactions and finite-size effects induce a tilt profile across the films. In particular we find that the tilt direction profile for the ferrielectric Sm-C ${ }_{\gamma}^{*}$ phase corresponds to a three-layer helix. Finally in thin films the incommensurate short-pitch helical structure of the $\mathrm{Sm}-\mathrm{C}_{\alpha}^{*}$ phase is suppressed by surface interactions and replaced by an anticlinic $\mathrm{Sm}-\mathrm{C}_{A}^{*}$-like structure.

\section{EXPERIMENT}

\section{A. Sample preparation}

The compound under study is R-1-methylheptyl-4-(4-ndodecyloxybiphenyl-4-carbonyloxy)-3-fluorobenzoate, abbreviated as 12F1M7. The mesomorphic and electro-optic properties of this material have been characterized both in bulk and in freely suspended films [18]. The molecular structure and the phase sequence with four different tilted smectic phases are shown in Fig. 1.

Freely suspended films were drawn in the Sm- $A$ phase using two different types of frames. For optical ellipsometry a rectangular variable-area frame was used of typically $5 \times 10 \mathrm{~mm}^{2}$. Requirements on the smectic films for $\mathrm{x}$-ray reflectivity are more stringent. Because of the large footprint at the small scattering angles, the films should be rather elongated. Moreover, the mosaicity, which is determined by the residual curvature of the film, should be as small as possible. For this reason films were spanned over a $10 \times 25 \mathrm{~mm}^{2}$ rect- angular hole with sharp edges made in a polished 2-mmthick glass plate. Nickel electrodes evaporated onto the glass were used to produce an aligning electric field along the short direction of the film. The films were mounted vertically in a temperature controlled two-stage oven and evacuated in order to prevent sample degradation and reduce air scattering. The films were equilibrated as described earlier [19].

\section{B. Optical ellipsometry}

The beam of a He-Ne laser passes through the film at an incident angle of $45^{\circ}$. Details of the set-up are presented in Ref. [20]. In short, using a null ellipsometer we determine the quantities $\Delta$ and $\Psi$ which describe the state of polarization of the transmitted light. The parameter $\Delta$ corresponds to the phase difference between the $p$ - and $s$-polarized components of the transmitted light. $\Psi$ is related to the amplitudes $T_{p, s}$ of the $p$ and $s$ components via $\tan \Psi=T_{p} / T_{s}$. The polarization of the incident light corresponds to $\Delta=0$ and $\Psi$ $=45^{\circ}$. The plane of incidence is determined by the film normal and the direction of the incident laser beam. A weak dc electric field $(8 \mathrm{~V} / \mathrm{cm})$ is applied perpendicular to this plane along the film. This field is large enough to align the net electric polarization of the film, while being too weak to distort the internal film structure.

In a conventional synclinic ferroelectric $\mathrm{Sm}-\mathrm{C}^{*}$ phase (helical pitch considerably larger than the film thickness) the director tilts either away $\left(\Delta_{+}\right)$from or towards $\left(\Delta_{-}\right)$the incident laser beam according to the polarity of the dc field. The difference is a measure of the tilt angle of the optical indicatrics of the film; hence in the Sm- $A$ phase $\Delta_{+}=\Delta_{-}$. In $\mathrm{Sm}-\mathrm{C}$ phases with anticlinic ordering and/or helical structures with a pitch comparable to or shorter than the film thickness, the values of $\Delta_{+}$and $\Delta_{-}$depend in a less straightforward way on the film structure. The only possibility is to guess a reasonable structure, to calculate the corresponding values of $\Delta_{+}$and $\Delta_{-}$, and then to compare calculated and measured values. The values of $\Delta_{+}$and $\Delta_{-}$can be calculated for arbitrary layered structures using the so-called $4 \times 4$ matrix method [21].

\section{X-ray reflectivity}

In a specular $\mathrm{x}$-ray reflectivity experiment an incident beam of wavelength $\lambda$ and wave number $k=2 \pi / \lambda$ is reflected at an interface. For elastic scattering, $\left|\mathbf{k}_{\mathbf{f}}\right|=\left|\mathbf{k}_{\mathbf{i}}\right|=k$, while the incident wave vector $\mathbf{k}_{\mathbf{i}}$, the reflected wave vector $\mathbf{k}_{\mathbf{f}}$ and the surface normal lie in the same (scattering) plane. Consequently, the resulting momentum transfer $\mathbf{q}=\mathbf{k}_{\mathbf{f}}-\mathbf{k}_{\mathbf{i}}$ is parallel to the surface normal $\left(|\mathbf{q}|=q_{z}=2 k \sin \alpha\right.$, where $\alpha$ is the incoming angle). In the case of a film, reflection also occurs at the second interface, leading to constructive or destructive interference in dependence of the incoming angle (Kiessig or interference fringes). The period of the fringes is inversely proportional to the film thickness $D$. In smectic films the internal periodic structure generates in addition finite-size Bragg-like peaks centered at $q_{z}=2 \pi \mathrm{m} / d$, where $d$ is the layer spacing and $m$ an integer. Thus the number of smectic layers $N=D / d$ can be determined unambiguously from these specular scans. 


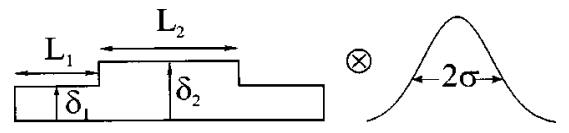

FIG. 2. Box model to represent the molecular form factor, which is convoluted with a Gaussian of width $2 \sigma$ to reconstruct the smectic density wave.

$\mathrm{X}$-ray reflectivity measurements were carried out at beamline BW2 of HASYLAB (DESY, Hamburg) at an energy of $8.6 \mathrm{keV}$. The experimental resolution was determined by slits upstream of the sample and in front of the detector as described in Ref. [22]. The angular resolution of the setup was $\Delta q_{z}=0.03 \mathrm{~nm}^{-1}$ full width at half maximum (FWHM) as measured by a detector scan in the scattering plane. In the direction along $q_{x}$ in the scattering plane we had $\Delta q_{x}$ $=0.004 \mathrm{~nm}^{-1}$. Out of the scattering plane the resolution was set wide in order to gain in intensity. Reflectivity measurements were also made at a home-built triple-axis diffractometer equipped with a Rigaku RU-300H generator operating at $18 \mathrm{~kW}$ power and utilizing $\mathrm{Cu} K_{\alpha}$ radiation. The incident beam was monochromatized and focused in the direction perpendicular to the scattering plane by a bent graphite (002) crystal. The resolution was chosen to be essentially the same as at BW2. Further details have been described elsewhere [19]. Specular scans probe the scattered intensity in reciprocal space along $q_{z}$ with $q_{x}$ equal to zero. The quality of the sample is monitored by measuring rocking curves at different $q_{z}$, varying $q_{x}$ by rotation of the sample. All curves have been background subtracted and corrected for geometrical effects as described earlier [19].

Information about the electron density distribution $\rho(z)$, including the phase at the interfaces, can be extracted by fitting the data to an iterative matrix solution of the Fresnel equations for the reflectivity of a multilayer system (Parratt algorithm), convoluted with the instrumental resolution [23]. The electron density $\rho_{e}$ is related to the refractive index $n$ $=1-\delta$ via the expression $\rho_{e}=2 \pi \delta /\left(\lambda^{2} r_{e}\right)$, where $r_{e}$ is the classic radius of the electron and $\delta \approx 4 \times 10^{-6}$ for organic materials at the $\mathrm{x}$-ray wavelengths used. The film is described by a succession of smectic layers of a certain thickness and electron density. To introduce the molecular form factor each of the layers is approximated by the boxlike function shown in Fig. 2. $L_{1}$ and $L_{2}$ represent the thickness corresponding to the core regions and to the aliphatic tails of the mesogens, respectively [19]. Smearing of the distribution of the molecular center of mass within the layers is taken into account by convolution with a Gaussian. Note that $d=L_{1}$ $+2 L_{2}$ in the bulk of an orthogonal phase like Sm- $A$. For tilted phases one must distinguish between the molecular form factor (which is now tilted with respect to the layer normal) and the layer form factor. The latter can be described as a "compressed" molecular form factor with $d_{i}=L_{1 i}$ $+2 L_{2 i}$. In the fitting procedure $L_{1,2 i}$ and $\sigma_{i}$ were left free for each smectic layer, taking the centrosymmetry of the density profile into account. In contrast $\delta_{1,2 i}$ was kept fixed for each particular set of data. In the absence of reliable measurements around the critical angle no absolute values of $\delta_{1}$ and

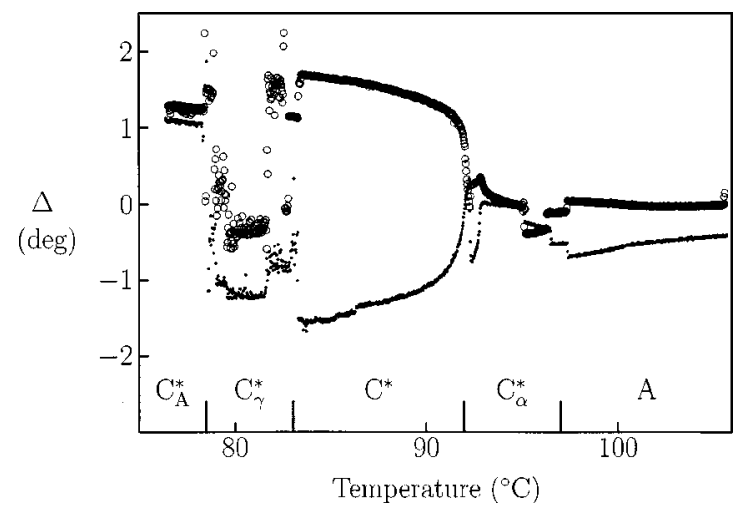

FIG. 3. Temperature dependence of the ellipsometric quantities $\Delta_{+}$(filled symbols) and $\Delta_{-}$(open symbols) for a 40-layer film of 12F1M7.

$\delta_{2}$ have been determined. Nevertheless, all calculations converged to $\delta_{2} / \delta_{1} \approx 1.4$.

\section{RESULTS}

Figure 3 shows ellipsometric results for a 40-layer film in which the five different regions corresponding to the various phases can easily be distinguished. At temperatures above $97^{\circ} \mathrm{C}$, where bulk samples show a Sm- $A$ phase, we measure, nevertheless, $\Delta_{+} \neq \Delta_{-}$. Evidently the surface layers tilt already at temperatures higher than the corresponding bulk transition $\left(T=92{ }^{\circ} \mathrm{C}\right)$. The $\mathrm{Sm}-\mathrm{C}_{\alpha}^{*}$ phase is observed in the range $97-92^{\circ} \mathrm{C}$ as indicated by the oscillating behavior of $\Delta_{+,-}$just above $92{ }^{\circ} \mathrm{C}[10]$. The conventional ferroelectric $\mathrm{Sm}-\mathrm{C}^{*}$ phase is seen between 92 and $83^{\circ} \mathrm{C}$, while the noisy $\Delta$ values between 83 and $78^{\circ} \mathrm{C}$ are typical for the $\mathrm{Sm}-\mathrm{C}_{\gamma}^{*}$ phase. Finally below $78^{\circ} \mathrm{C}$ the antiferroelectric $\mathrm{Sm}-\mathrm{C}_{A}^{*}$ phase is stable.

Figure 4 gives specular x-ray scans for a relatively thick freely suspended film (about 150 layers), which is typical for the "bulk" structure of the various chiral Sm-C phases. Two orders of Bragg peaks result from the smectic layering. The intensity of the second harmonic is about two orders of mag-

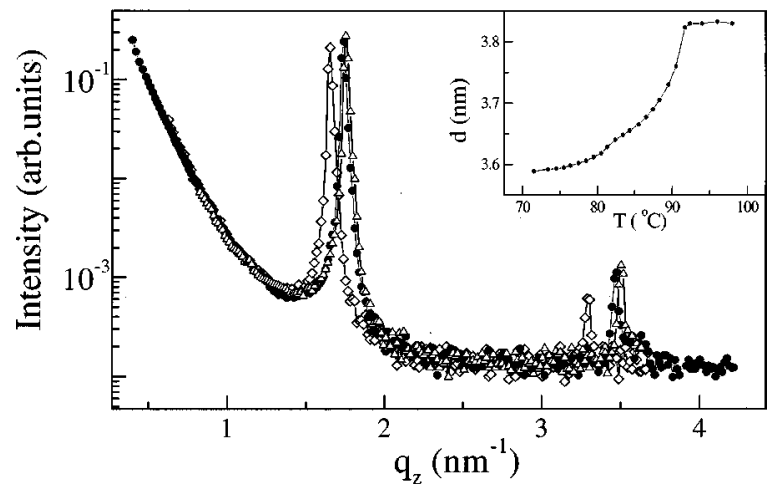

FIG. 4. Specular $\mathrm{x}$-ray reflectivity for a thick $(\approx 150$ layers $)$ freely suspended smectic film in the $\mathrm{Sm}-A\left(T=97^{\circ} \mathrm{C}\right.$, open diamonds $), \mathrm{Sm}-C_{\gamma}^{*}\left(T=81^{\circ} \mathrm{C}\right.$, triangles $)$ and $\mathrm{Sm}-\mathrm{C}_{A}^{*}\left(T=73^{\circ} \mathrm{C}\right.$, filled squares) phases. The inset shows the temperature dependence of the layer spacing $d$ as determined from the Bragg position. 
nitude smaller than that of the fundamental harmonic, which is quite usual for soft, layered smectic materials [24]. Starting from $T \approx 92{ }^{\circ} \mathrm{C}$ the Bragg positions shift to larger $q_{z}$ values upon decreasing the temperature, which signals a transition to tilted smectic phases. The full temperature variation of the layer spacing $d$ is shown in the inset of Fig. 4 . We note a rather continuous decrease of $d$ (increase of the tilt angle); the cusp at $T \approx 83^{\circ} \mathrm{C}$ probably indicates the transition to the ferroelectric $\mathrm{Sm}-\mathrm{C}_{\gamma}^{*}$ phase. We searched explicitly for evidence of possible superlattices in the system, which should show up as subharmonics of the single layer spacing. However, in all the variants of the $\mathrm{Sm}-\mathrm{C}$ phase only the monolayer periodicity was observed (compare Fig. 4).

In Fig. 5(a) we present specular x-ray reflectivity results of a six-layer film in the various $\mathrm{Sm}-\mathrm{C}$ phases. The curves show Kiessig fringes that correspond to the total film thickness, and first- and second-order finite-size broadened Bragg peaks. The films were remarkably uniform (mosaicity $\approx 0.01^{\circ}$ FWHM) and stable as confirmed by monitoring rocking curves during the experiment. The intensity in the region just after the first and second Bragg peak is most sensitive to the actual electron density distribution [19]. A first try to fit the data with a constant layer spacing throughout the film failed immediately: the only possibility is a layer thickness profile across the film. The resulting modulated density distribution over the film is displayed in Fig. 5(b). The parameters of the best fit to the full experimental data using the model described in the previous section are given in Table I. In a simple hard-rod approximation the tilt couples to the layer spacing via $d_{i}=d_{A} \cos \theta_{i}$, where $d_{A}$ is the layer spacing in the bulk Sm- $A$ phase. The corresponding tilt magnitude profile $\theta(z)$ for the six-layer film is presented in Fig. 6. The tilt is largest for the surface layers and decreases further into the interior of the film. In the range of stability of the $\mathrm{Sm}-\mathrm{C}_{\alpha}^{*}$ phase the tilt magnitude profile is flat in the interior, while in the all other tilted phases there is a continuous change of $\theta(z)$. In these latter cases the tilt profile across the film can be well fitted by $\theta(z)$ $=\theta_{\text {surf }}[\cosh (2 z-D) / 2 \xi] / \cosh (D / 2 \xi)$, where $\theta_{\text {surf }}$ is the value of the tilt angle at the free surfaces $(z=0, D)[25,26]$. From Table I we note also a Gaussian width (fluctuation) profile across the film. At the surface the fluctuations are quenched by the surface tension in agreement with the hydrodynamic theory of smectic layer displacement fluctuations [27].

Unfortunately, in ellipsometry thin films always lead to noisy data and we could not obtain useful results from a six-layer film of $12 \mathrm{~F} 1 \mathrm{M} 7$. The values of $\Delta_{+}$and $\Delta_{-}$for the thinnest film which gave reproducible results (10 layers) are shown in Fig. 7(a). The five different phases which are easily observed in the 40-layer film (Fig. 3), are still discernible. To model the essential experimental features we extrapolated for each of the various $\mathrm{Sm}-\mathrm{C}^{*}$ phases the tilt magnitude profiles of the six-layer film to the ten-layer film. For the Sm- $A$ phase (with tilted surface layers), the $\mathrm{Sm}-\mathrm{C}^{*}$, the $\mathrm{Sm}-\mathrm{C}_{\gamma}^{*}$ and the $\mathrm{Sm}-\mathrm{C}_{A}^{*}$ phases the interpolated continuous cosh-shaped tilt profiles were used. For the $\mathrm{Sm}-\mathrm{C}_{\alpha}^{*}$ phase a flat profile (constant $\theta$ ) was used for the eight interior layers and a different value for the two surface layers. In this way we can
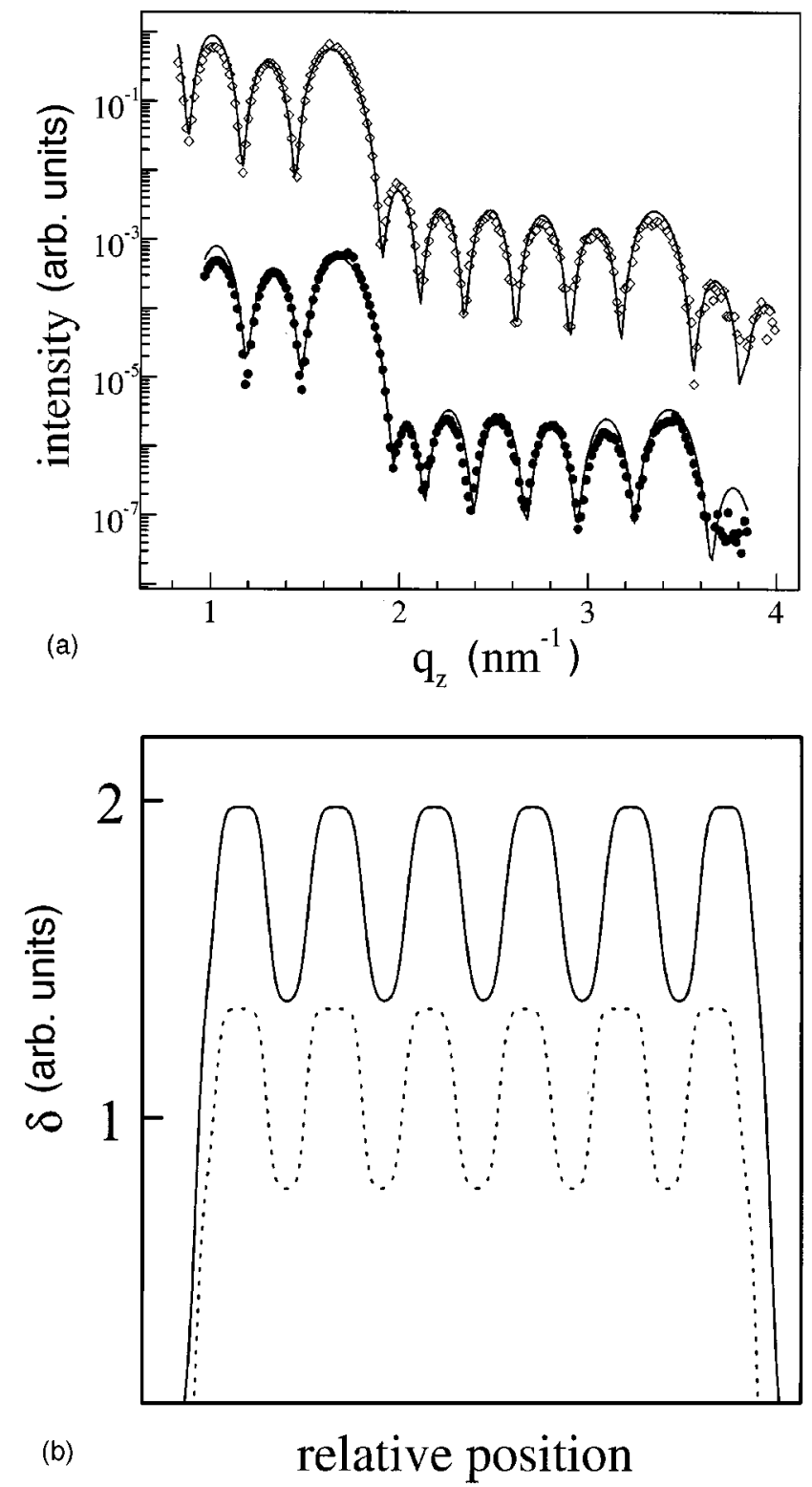

FIG. 5. Specular x-ray reflectivity of a six-layer film with best fit to the data (a) and the associated electron density profile (b). Data taken in $\mathrm{Sm}-\mathrm{C}_{\alpha}^{*}$ phase $\left(T=94^{\circ} \mathrm{C}\right.$, filled dots $)$ and $\mathrm{Sm}-\mathrm{C}_{\gamma}^{*}(T$ $=81{ }^{\circ} \mathrm{C}$, diamonds). Curves have been shifted for clarity.

calculate $\Delta_{+}$and $\Delta_{-}$for a ten-layer model film in which both the tilt magnitude and direction are appropriately adjusted for each layer. The resulting values of $\Delta_{+}$and $\Delta_{-}$are shown in Fig. 7(b) and reproduce the essential features of Fig. 7(a) nicely. Figure 8 gives the underlying model tilt magnitude and tilt direction profiles. They will be discussed more extensively in the next section.

\section{DISCUSSION}

At first sight x-ray diffraction and reflectivity, though powerful tools in determining the structure and interfacial properties of thin film molecular assemblies, are not expected to distinguish between the various chiral Sm-C struc- 
TABLE I. Fitting parameters for the x-ray reflectivity of the six-layer film (compare Fig. 5) in the various types of chiral Sm-C phase. Typical error bars are $0.1 \mathrm{~nm}$ for $d$ and $0.05 \mathrm{~nm}$ for $\sigma$.

\begin{tabular}{lcccc}
\hline \hline layer number & & 1,6 & 2,5 & 3,4 \\
\hline$T=75^{\circ} \mathrm{C}$ & thickness $d(\mathrm{~nm})$ & 3.43 & 3.53 & 3.60 \\
& Fluctuations $\sigma(\mathrm{nm})$ & 0.22 & 0.25 & 0.25 \\
$T=81^{\circ} \mathrm{C}$ & $d(\mathrm{~nm})$ & 3.47 & 3.56 & 3.61 \\
& $\sigma(\mathrm{nm})$ & 0.20 & 0.25 & 0.25 \\
$T=88^{\circ} \mathrm{C}$ & $d(\mathrm{~nm})$ & 3.46 & 3.59 & 3.64 \\
& $\sigma(\mathrm{nm})$ & 0.19 & 0.26 & 0.29 \\
$T=94^{\circ} \mathrm{C}$ & $d(\mathrm{~nm})$ & 3.43 & 3.72 & 3.71 \\
& $\sigma(\mathrm{nm})$ & 0.25 & 0.29 & 0.30 \\
$T=96^{\circ} \mathrm{C}$ & $d(\mathrm{~nm})$ & 3.43 & 3.75 & 3.76 \\
& $\sigma(\mathrm{nm})$ & 0.29 & 0.35 & 0.38 \\
\hline \hline
\end{tabular}

tures. The crucial point is that conventional x-ray techniques are only sensitive to electron density modulations, because the corresponding susceptibility is a scalar quantity. This situation is changed in the case of resonant x-ray scattering, where the susceptibility becomes a tensor [8]. If we consider the ferroelectric Sm-C* or antiferroelectric $\mathrm{Sm}-\mathrm{C}_{A}^{*}$ phase it is clear that the electron density projected along $\mathbf{z}$ is the same for each layer. These phases have either synclinic $\left(\mathrm{Sm}-\mathrm{C}^{*}\right)$ or anticlinic $\left(\mathrm{Sm}-\mathrm{C}_{A}^{*}\right)$ interfaces and they differ from one another only by symmetry elements such as glide planes or $2{ }_{1}$ screw axes along $\mathbf{z}$. As the smectic phases under study have a liquidlike in-plane order, in reciprocal space in the wide-angle region only a broad diffuse halo is observed. Consequently the symmetry elements mentioned cannot lead to specific extinction rules as is the case for higher ordered structures. However, the situation may be different in the case of ferroelectric Sm-C $\mathrm{C}_{\gamma}^{*}$ phases. For a structure corresponding to a devil's staircase there are two different kinds of interfaces: between the synclinic layers and between anticlinic layers [Fig. 9(a)]. The steric requirements or other types of local interaction (for example dipoles [28]) that

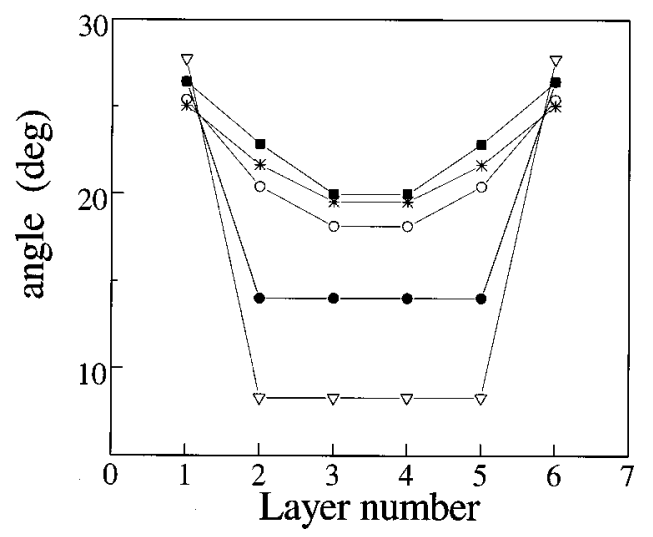

FIG. 6. Tilt magnitude profiles in the various tilted $\mathrm{Sm}-\mathrm{C}^{*}$ phases as determined from specular x-ray reflectivity of a six-layer film: Sm-C ${ }_{\alpha}^{*}\left(T=96{ }^{\circ} \mathrm{C}\right.$, open triangles, $T=94^{\circ} \mathrm{C}$, filled dots $)$, Sm-C ${ }^{*}\left(T=88^{\circ} \mathrm{C}\right.$, open dots $), \mathrm{Sm}_{-} \mathrm{C}_{\gamma}^{*}\left(T=81^{\circ} \mathrm{C}\right.$, asterisks $)$, $\mathrm{Sm}-\mathrm{C}_{A}^{*}\left(T=75^{\circ} \mathrm{C}\right.$, filled squares $)$.
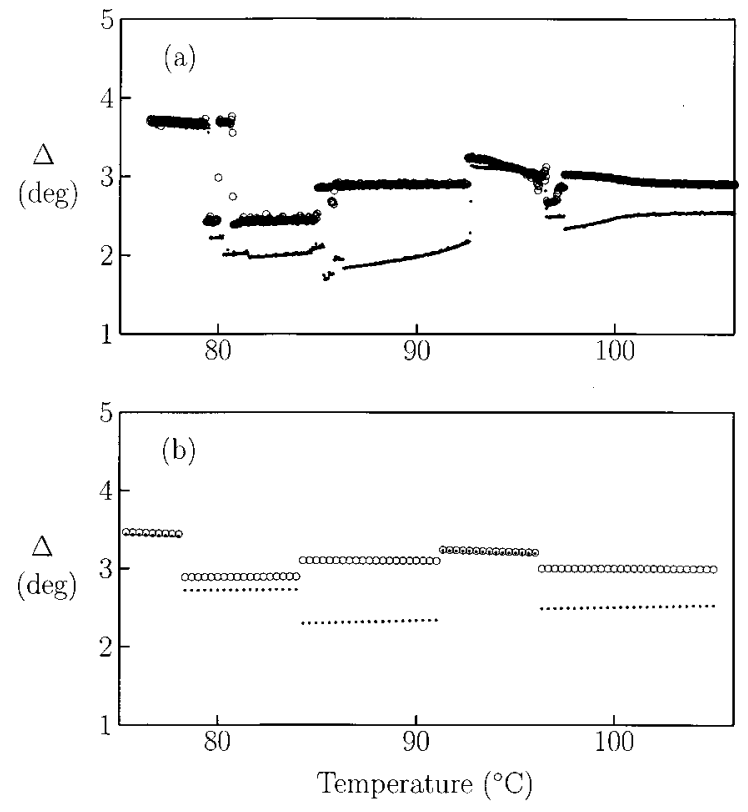

FIG. 7. Measured (a) and calculated values (b) of the ellipsometric quantities $\Delta_{+}$(filled symbols) and $\Delta_{-}$(open symbols) of a ten-layer film of $12 \mathrm{~F} 1 \mathrm{M} 7$.

could stabilize anticlinic ordering are likely to be different in the two cases, and this asymmetry might be measurable in specular reflectivity. If $q_{1}$ corresponds to a single layer spacing, then superlattices at $q_{1} / 3$ or a $q_{1} / 4$ should be observable. In principle this could allow discriminating between ferriand ferro- antiferroelectric states in chiral smectics. The $q_{z}$ dependence of the thermal layer displacement fluctuations could also be different in ferro- and ferroelectric phases. Our
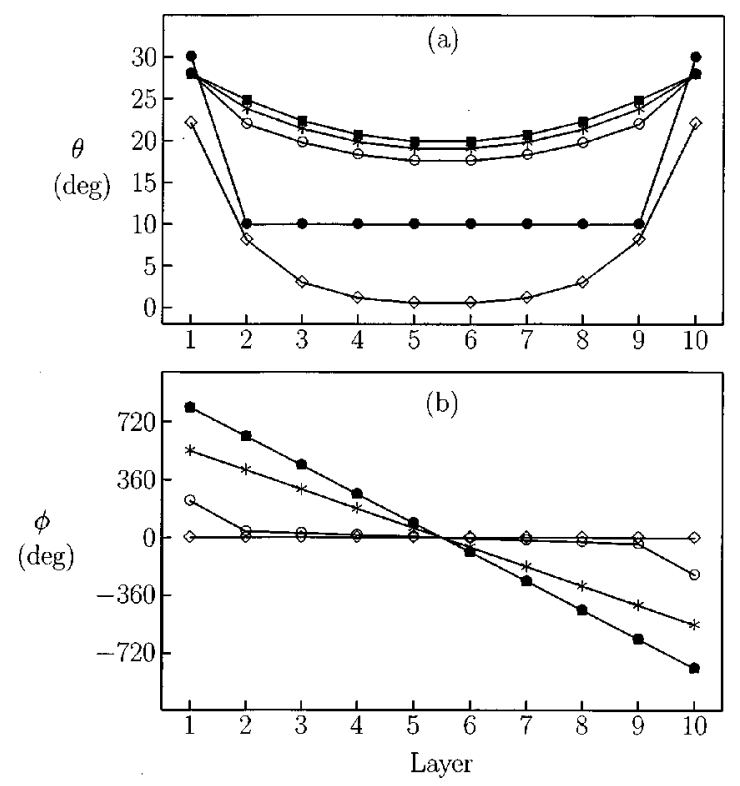

FIG. 8. (a) Tilt magnitude profiles $\theta(z)$ and (b) tilt direction profiles $\phi(z)$ of a ten-layer model film, as used for the calculation of $\Delta_{+}$and $\Delta_{-}$of Fig. 7(b). Sm- $A$ with tilted surface layers (open diamonds), $\mathrm{Sm}-\mathrm{C}_{\alpha}^{*}$ (filled dots), $\mathrm{Sm}-\mathrm{C}^{*}$ (open dots), $\mathrm{Sm}-\mathrm{C}_{\gamma}^{*}$ (asterisks), $\mathrm{Sm}-\mathrm{C}_{A}^{*}$ (filled squares). 


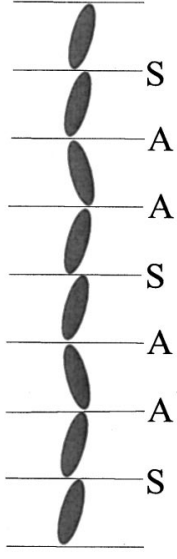

a)

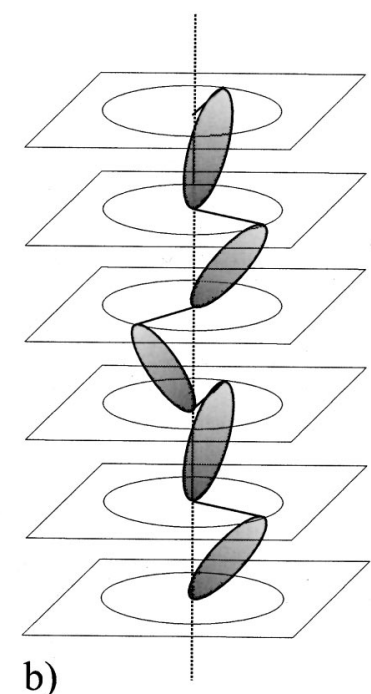

b)
FIG. 9. Interlayer structure of some possible ferrielectric $\mathrm{Sm}-\mathrm{C}_{\gamma}^{*}$ models. (a) Sequence of synclinic $(S)$ and anticlinic $(A)$ layer interfaces according to a devil's staircase; (b) Discrete variations of the azimuthal angle as given in the clock model.

x-ray measurements on thick freely suspended films of 12F1M7 show no indications of subharmonics of $q_{1}$. Similar bulk results have been reported for another type of chiral compound exhibiting two different ferroelectric phases in Ref. [29]. There is also no significant difference in specular reflectivity of the ferro-, antiferro-, and ferroelectric phases. This result is to be expected for the antiferroelectric $\left(\mathrm{Sm}-\mathrm{C}_{A}^{*}\right)$ phase. For the ferrielectric $\left(\mathrm{Sm}-\mathrm{C}_{\gamma}^{*}\right)$ phase it signifies that the layer interfaces are all identical, thus favoring the clock models for the tilt direction distribution [Fig. 9(b)].

Contrary to conventional x-ray reflectivity, optical ellipsometry is sensitive to both the tilt magnitude and tilt azimuthal distribution across the film. This is due to the fact that for the optical range of wavelengths the electric field interacts with the liquid crystal director via the local dielectric susceptibility tensor. However, because of the limited range of experimental variables, ellipsometry suffers strongly from the inversion problem, i.e., different refractive index profiles may lead to similar ellipsometric characteristics. In principle the density profiles obtained via x-ray reflectivity might also not be unique, because they are obtained by fitting and not by a direct inversion of data. This is related to the well known "phase problem" of x-ray diffraction. However, for systems with a 1D density modulation the density profile can be fully reconstructed from a single $\mathrm{x}$-ray reflectivity experiment if certain conditions are fulfilled [15]. It requires that the phase of the complex reflection coefficient is completely determined by its modulus, i.e., by the measured reflectivity. For example, in freely suspended smectic films the phase of the sinelike density modulation [Fig. 5(b)] is fully determined by the density termination at the free interface $[30,31]$. It may be lower or higher than the density in the middle of the smectic top layer depending on the structure of the constituent molecules and their anchoring properties. For conventional mesogenic molecules with hydrocarbon tails the smectic density shows a minimum at the liquid crystal-air interface. This leads to an enhancement and reduction of the scattered intensity at the low- $q$ and high- $q$ side of the first Bragg peak, respectively. In the case of a highdensity termination at the free interface this asymmetry in the intensity around the first Bragg peak reverses [19]. In conclusion, the density profile in freely suspended smectic films can be unambiguously reconstructed from x-ray reflectivity measurements. Subsequently we have used the resulting tilt magnitude profiles as input for modeling the ellipsometric data, which then in turn leads to precise tilt direction profiles.

We now come to a further discussion of the profiles given in Fig. 8(b). In the Sm- $A$ phase with tilted surface layers we find that the tilt direction is in all layers perpendicular to the applied field as expected. For the $\mathrm{Sm}_{-} \mathrm{C}_{\alpha}^{*}$ phase the ellipsometric data are best described by a tilt direction profile corresponding to a layer-by-layer alternation of the tilt direction. This double-layer helix differs from the $\mathrm{Sm}-\mathrm{C}_{\alpha}^{*}$ bulk structure, which is characterized by a helix with a temperature dependent pitch in the range from 5 to 40 layers $[8,10,32]$. Contrary to other $\mathrm{Sm}-\mathrm{C}^{*}$ phases the tilt magnitude profile in this temperature range does not have the shape of a cosh: the profile is flat in the interior of the film and the tilt angle increases abruptly in the two surface layers. Such a behavior is very different from the continuous penetration of tilt from the surface to the interior of a film upon approaching a $\mathrm{Sm}-A-\mathrm{Sm}-\mathrm{C}$ transition. In fact there is no pretransitional growth of the $\mathrm{Sm}-\mathrm{C}_{\alpha}^{*}$ phase in $12 \mathrm{~F} 1 \mathrm{M} 7$ when approached through the $\mathrm{Sm}-A$ from higher temperatures. Instead in that situation a surface-induced $\mathrm{Sm}-\mathrm{C}^{*}$ phase is observed [10]. We conclude that in thin films like the ten-layer one, the bulk helix structure of the $S m-C_{\alpha}^{*}$ phase is suppressed by surface interactions. Instead a $\mathrm{Sm}-\mathrm{C}_{A}^{*}$-like structure with anticlinic layer-by-layer alternation of the tilt direction is formed. Thus, surface interactions can modify not only the tilt magnitude but also the tilt direction. In the $\mathrm{Sm}-\mathrm{C}^{*}$ phase the ellipsometric data are consistent with a 33-layer helix with an additional change in the tilt direction in the two surface layers as described in Ref. [33]. The tilt directions in the surface layers at both sides of a film are correlated and correspond to anticlinic tilt structures in agreement with recent observations $[11,26,33,34]$. For the $\mathrm{Sm}_{-} \mathrm{C}_{\gamma}^{*}$ phase the tilt direction profile in the model film was adjusted to a three-layer helix, as indicated by resonant x-ray diffraction [8]. Using in addition the tilt magnitude profile derived from $\mathrm{x}$-ray reflectivity, this results in a fair reproduction of the measured ellipsometry data. These results can be considered as independent evidence for the clock model of the $\mathrm{Sm}-\mathrm{C}_{\gamma}^{*}$ phase. For the $\mathrm{Sm}-\mathrm{C}_{A}^{*}$ phase we find the expected layer-by-layer tilt alternation (two-layer helix).

Finally we note from Fig. 7 that in narrow temperature intervals in the vicinity of the phase transitions the ellipsometric data give values of $\Delta_{+}$and $\Delta_{-}$that clearly differ from those well within the corresponding phase ranges. This could indicate that the transitions between the different phases take place in two or three steps, at least for a ten-layer film. 


\section{CONCLUSIONS}

We have used x-ray reflectivity and optical ellipsometry to characterize the structure of freely suspended films of a chiral liquid crystal possessing a sequence of five Sm-C $C^{*}$-type phases. By combining both methods detailed interlayer structural information is obtained regarding both the tilt magnitude and tilt direction profiles in thin films. The density profiles can be unambiguously reconstructed from the $\mathrm{x}$-ray reflectivity measurements and have been converted to tilt magnitude profiles. Using these as input to model the average optical properties obtained by ellipsometry, also tilt direction profiles are obtained, and realistic models can be elaborated for the various types of phase in Sm-C* films. In general the surface layers are more tilted than the interior layers due to surface interactions and finite-size effects.

Within the accuracy of our measurements there are no indications of subharmonics of the single layer periodicity in the various Sm-C* phases, in agreement with "clock" models for the tilt direction distribution. Comparison of the measured ellipsometric quantities with calculated values resulting from model films, provides evidence of a three-layer helix for the ferrielectric Sm-C $\mathrm{C}_{\gamma}^{*}$ phase. Further for thin films (ten-layer and less) the surface interactions suppress the short-pitch helical structure of the $\mathrm{Sm}-\mathrm{C}_{\alpha}^{*}$ phase. It is replaced by the $\mathrm{Sm}-\mathrm{C}_{A}^{*}$-like structure with anticlinic layer-bylayer alternation of the tilt direction within the film.

\section{ACKNOWLEDGMENTS}

The authors wish to thank M. Cepic, V. Dmitrienko, V. Lorman, and S. Pikin for valuable discussions. They are grateful to G. Wong for his participation at the initial stage of the project. Work at HASYLAB was supported by Contract No. ERBFMGECT950059 of the European Community. This work is part of the research program of the "Stichting voor Fundamenteel Onderzoek der Materie (FOM),', which is financially supported by the "Nederlandse Organisatie voor Wetenschappelijk Onderzoek (NWO).' Part of this work was supported by the Deutsche Forschungsgemeinschaft (Grant No. Ba 1048/5) and the Fonds der Chemischen Industrie.
[1] See, for some recent reviews, J. W. Goodby, R. Blinc, N. A. Clark, S. T. Lagerwall, M. A. Osipov, S. A. Pikin, T. Sakurai, K. Yoshino, and B. Zeks, Ferroelectric Liquid Crystals. Principles, Properties and Applications (Gordon \& Breach, Philadelphia, 1991); S. T. Lagerwall, Ferroelectric and Antiferroelectric Liquid Crystals (Wiley-VCH, Weinheim, 1999).

[2] A. Fukuda, Y. Takanishi, T. Isozaki, K. Ishikawa, and H. Takezoe, J. Mater. Chem. 4, 997 (1994), and references therein.

[3] R. B. Meyer, L. Liebert, L. Strzelecki, and P. Keller, J. Phys. (France) Lett. 36, L69 (1975).

[4] A. D. L. Chandani, E. Gorecka, Y. Ouchi, H. Takezoe, and A. Fukuda, Jpn. J. Appl. Phys., Part 2 28, L1265 (1989).

[5] M. Skarabot, M. Cepic, B. Zeks, R. Blinc, G. Heppke, A. V. Kityk, and I. Musevic, Phys. Rev. E 58, 575 (1998).

[6] Y. Takanishi, K. Hiraoka, V. K. Agrawal, H. Takezoe, A. Fukuda, and M. Matsushita, Jpn. J. Appl. Phys., Part 1 30, 2023 (1991); T. Isozaki, K. Hiraoka, Y. Takanishi, H. Takezoe, A. Fukuda, Y. Suzuki, and I. Kawamura, Liq. Cryst. 12, 59 (1992).

[7] M. Cepic and B. Zeks, Mol. Cryst. Liq. Cryst. Sci. Technol., Sect. A 263, 61 (1995); V. L. Lorman, ibid. 262, 437 (1995); S. A. Pikin, S. Hiller, and W. Haase, ibid. 262, 425 (1995); V. L. Lorman, Liq. Cryst. 20, 267 (1996); A. Roy and N. Madhusudana, Europhys. Lett. 36, 221 (1996).

[8] P. Mach, R. Pindak, A.-M. Levelut, P. Barois, H. T. Nguyen, C. C. Huang, and L. Furenlid, Phys. Rev. Lett. 81, 1015 (1998); P. Mach, R. Pindak, A.-M. Levelut, P. Barois, H. T. Nguyen, H. Baltes, M. Hird, K. Toyne, A. Seed, J. W. Goodby, C. C. Huang, and L. Furenlid, Phys. Rev. E 60, 6793 (1999).

[9] P. M. Johnson, D. A. Olson, S. Pankratz, T. Nguyen, J. W. Goodby, M. Hird, and C. C. Huang, Phys. Rev. Lett. 84, 4870 (2000).
[10] D. Schlauf, Ch. Bahr, and H. T. Nguyen, Phys. Rev. E 60, 6816 (1999).

[11] P. M. Johnson, S. Pankratz, P. Mach, H. T. Nguyen, and C. C. Huang, Phys. Rev. Lett. 83, 4073 (1999).

[12] B. M. Ocko, A. Breslau, P. S. Pershan, J. Als-Nielsen, and M. Deutsch, Phys. Rev. Lett. 57, 94 (1986); E. F. Gramsbergen, W. H. de Jeu, and J. Als-Nielsen, J. Phys. (Paris) 47, 711 (1986); E. B. Sirota, P. S. Pershan, S. Amador, and L. B. Sorensen, Phys. Rev. A 35, 2283 (1987); B. D. Swanson, H. Stragier, D. Tweet, and L. B. Sorensen, Phys. Rev. Lett. 62, 909 (1989); T. Stoebe, R. Geer, C. C. Huang, and J. W. Goodby, ibid. 69, 2090 (1992); C. Y. Chao, S. W. Hui, J. E. Maclennan, C. F. Chou, and J. T. Ho, ibid. 78, 2581 (1997).

[13] See, for some reviews, P. Pieranski et al., Physica A 194, 364 (1993); C. Bahr, Int. J. Mod. Phys. B 8, 3051 (1994); T. Stoebe and C. C. Huang, ibid. 9, 2285 (1995); A. A. Sonin, Freely Suspended Liquid Crystalline Films (Wiley, Chichester, 1998).

[14] S. Heinekamp, R. A. Pelcovits, E. Fontes, E. Y. Chen, R. Pindak, and R. B. Meyer, Phys. Rev. Lett. 52, 1017 (1984); S. M. Amador and P. S. Pershan, Phys. Rev. A 41, 4326 (1990); Ch. Bahr, C. J. Booth, D. Fliegner, and J. W. Goodby, Phys. Rev. Lett. 77, 1083 (1996).

[15] M. Tolan, X-ray Scattering from Soft Matter Thin Films (Springer, Berlin, 1999); X-ray and Neutron Reflectivity: Principles and Applications, edited by J. Daillant and A. Gibaud (Springer, Berlin, 2000).

[16] R. R. M. A. Azzam and N. M. Bashara, Ellipsometry and Polarized Light (North-Holland, Amsterdam, 1989).

[17] Ch. Bahr and D. Fliegner, Phys. Rev. Lett. 70, 1842 (1993).

[18] Ch. Bahr, D. Fliegner, C. J. Booth, and J. W. Goodby, Europhys. Lett. 26, 539 (1994); Phys. Rev. E 51, R3823 (1995).

[19] E. A. L. Mol, J. D. Shindler, A. N. Shalaginov, and W. H. de Jeu, Phys. Rev. E 54, 536 (1996); A. Fera, B. I. Ostrovskii, D. 
Sentenac, I. Samoilenko, and W. H. de Jeu, ibid. 60, R5033 (1999).

[20] Ch. Bahr and D. Fliegner, Phys. Rev. A 46, 7657 (1992).

[21] D. W. Berreman, J. Opt. Soc. Am. 62, 502 (1972).

[22] D. Sentenac, A. Shalaginov, A. Fera, and W. H. de Jeu, J. Appl. Crystallogr. 33, 130 (2000); D. Sentenac, A. Fera, R. Opitz, B. I. Ostrovskii, O. Bunk, and W. H. de Jeu, Physica B 283, 232 (2000).

[23] L. G. Parrat, Phys. Rev. 95, 359 (1954); I. I. Samoilenko, O. V. Konovalov, L. A. Feigin, B. M. Shchedrin, and L. G. Yanusova, Crystallogr. Rep. 44, 310 (1999).

[24] B. I. Ostrovskii, in Structure and Bonding, Liquid Crystals I, edited by D. M. P. Mingos (Springer, New York-Heidelberg, 1999), Vol. 94, p. 199.

[25] K. Binder, in Phase Transitions and Critical Phenomena, edited by C. Domb and J. L. Lebowitz (Academic, London, 1986), Vol. 8, p. 2. The original meaning of $\xi$ is the correlation length that determines the growth of the thickness of an ordered surface layer on bulk material.

[26] P. O. Andreeva, V. K. Dolganov, C. Gors, R. Fouret, and E. I. Kats, Phys. Rev. E 59, 4143 (1999).

[27] R. Holyst, Phys. Rev. A 44, 3692 (1991); A. N. Shalaginov and V. P. Romanov, Phys. Rev. E 48, 1073 (1993). The layer displacement fluctuations are quenched at the free surface of smectics if $\gamma>(K B)^{1 / 2}$, where $\gamma$ is a surface tension and $\mathrm{K}$ and $\mathrm{B}$ the elastic constants for bending and compression of the layers, respectively. A quenched situation is usual for mesogenic molecules with terminal hydrocarbon tails.

[28] Y. Takanishi, K. Hiraoka, V. K. Agarwal, H. Takezoe, A. Fukuda, and M. Matsushita, Jpn. J. Appl. Phys., Part 1 30, 2023 (1991); K. Miyachi, J. Matsushima, Y. Takanishi, H. Takezoe, and A. Fukuda, Phys. Rev. E 52, R2153 (1995).

[29] P. Cluzeau, P. Barois, H. T. Nguyen, and C. Destrade, Eur. Phys. J. B 3, 73 (1999).

[30] S. Gierlotka, P. Lambooy, and W. H. de Jeu, Europhys. Lett. 12, 341 (1990).

[31] D. J. Tweet, R. Holyst, B. D. Swanson, H. Stragier, and L. B. Sorensen, Phys. Rev. Lett. 65, 2157 (1990). To the best of our knowledge this is the only paper in which tilt magnitude profiles have been determined from $\mathrm{x}$-ray reflectivity in Sm-C freely suspended films.

[32] This temperature dependence causes the oscillatory behavior of $\Delta_{+}$and $\Delta_{-}$[10], which is also clearly seen in the 40-layer film of Fig. 3.

[33] D. Schlauf and Ch. Bahr, Phys. Rev. E 57, R1235 (1998).

[34] P. O. Andreeva, V. K. Dolganov, and K. P. Meletov, Pis'ma Zh. Eksp. Teor. Fiz. 66, 414 (1997) [JETP Lett. 66, 442 (1997)]; D. Schlauf, Ch. Bahr, V. K. Dolganov, and J. W. Goodby, Eur. Phys. J. B 9, 461 (1999); D. R. Link, G. Natale, N. A. Clark, J. E. Maclennan, M. Walsh, S. S. Keast, and M. E. Neubert, Phys. Rev. Lett. 82, 2508 (1999). 